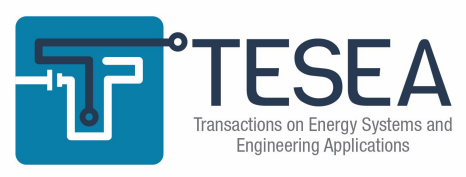

Article

\title{
A Comparative Study of Signal Analysis Methods Applied in the Detection of Instantaneous Frequency ${ }^{*}$
}

\author{
Maximiliano Bueno-Lopez ${ }^{1 \bowtie(D)}$ and J. Mauricio Sanabria-Villamizar ${ }^{2}$ \\ 1 Departamento de Electrónica, Instrumentación y Control, Universidad del Cauca. Calle 5 No. 4-70, Popayán, \\ Cauca, Colombia;mbuenol@unicauca.edu.co \\ 2 Programa de Ingeniería Eléctrica, Universidad de La Salle. Carrera 2 No. 10 - 70, Bogotá, Colombia; \\ jsanabria16@unisalle.edu.co

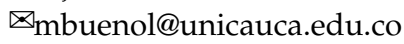

Received: 11 August 2020; Accepted: 5 September 2020; Published: 16 December 2020

\begin{abstract}
The smart grid concept is being applied more and more frequently and this is due to the need to integrate all the components that are part of power systems today, starting from generation units, storage systems, communications and connected loads. Non-linear and non-stationary signals have been obtained in this type of systems, which have high penetration of non-conventional energy sources (NCSRE) and non-linear loads. The power quality criterion has had to be adapted to the new conditions of the electrical systems and this has led to the need to search for new analysis methodologies for the acquired signals. In this article we present a review on non-linear and non-stationary signal analysis methods in electrical systems with high NCSRE penetration. To this end we explore the application of the Hilbert-Huang Transform (HHT), Wavelet Transform (WT) and Wigner-Ville Distribution (WVD), exposing each of the advantages and disadvantages of these methods. To validate the methodology, we have selected some synthetic signals that adequately describe the typical behaviors in these systems.
\end{abstract}

Keywords: Power Quality; Empirical Mode Decomposition; Instantaneous Frequency; Hilbert-Huang Transform; Wavelet Transform.

Resumen: El concepto de red inteligente se viene aplicando cada vez con mayor frecuencia y esto se debe a la necesidad de integrar todos los componentes que hacen parte de los sistemas de potencia en la actualidad, partiendo de las unidades de generación, sistemas de almacenamiento, comunicaciones y cargas. Señales no lineales y no estacionarias han sido obtenidas en este tipo de sistemas, los cuales poseen alta penetración de fuentes no convencionales de energía (FNCER) y cargas no lineales. El criterio de calidad de potencia se ha tenido que adaptar a las nuevas condiciones de los sistemas eléctricos y esto ha llevado a la necesidad de buscar nuevas metodologías de análisis de las señales adquiridas. En este artículo presentamos una revisión sobre los métodos de análisis de señales no lineales y no estacionarias en sistemas eléctricos con alta penetración de FNCER. Para este fin exploramos la aplicación de la Transformada de Hilbert-Huang (HHT), Transformada Wavelet (WT) y Distribución de Wigner-Ville (WVD), exponiendo cada una de las ventajas y desventajas de estos métodos. Hemos seleccionado para

${ }^{*}$ How to cite this article: Bueno-López, M., Sanabria Villamizar, J. (2020). A Comparative Study of Signal Analysis Methods Applied in the Detection of Instantaneous Frequency. Transactions on Energy Systems and Engineering Applications, 1(1): 1-11. DOI: 10.32397/tesea.vol1.n1.1 ISSN: 2745-0120 
validar la metodología algunas señales sintéticas que describen de manera adecuada los comportamientos típicos en los sistemas de potencia modernos.

Palabras claves: Calidad de Potencia; Descomposición Empírica de Modos; Frecuencia instantánea; Transformada de Hilbert-Huang; Transformada Wavelet.

\section{Introducción}

La llegada de nuevas tecnologías a los sistemas de potencia tales como las Fuentes no Convencionales de Energías Renovables (FNCER), las cargas no lineales y los dispositivos electrónicos, han causado problemas en la forma de la onda transmitida y esto ha generado la necesidad de desarrollar nuevas metodologías para el análisis de señales con estas características (Golpîra and Messina 2018; Naderi et al. 2020). Una buena calidad en el sistema de potencia asegura un suministro de energía con altos niveles de calidad y confiabilidad para el correcto funcionamiento de las cargas conectadas, los cuales están manejando procesos críticos y de gran importancia para los usuarios (Liu et al. 2015; Naderi et al. 2020).

Las alteraciones de la calidad de la potencia pueden clasificarse en dos categorías, estas son variaciones y eventos. Las variaciones son perturbaciones que tienen un efecto en cada ciclo, como armónicos o desequilibrios de voltaje. Los eventos son perturbaciones que pueden durar desde una fracción de un ciclo hasta varios de estos, y luego pueden no repetirse durante varias horas o días (Fan and Liu 2012; Kumar and Zare 2016). Los problemas más comunes de calidad de energía son la caída de tensión, los armónicos y los transitorios de conmutación. En su mayoría, estos son causados por la naturaleza intermitente de las generaciones de energía no convencionales, transitorios debido a la conmutación de capacitores y grandes alimentadores de carga (Anzalchi et al. 2019; Kavitha and Subramanian 2017).

En los sistemas de potencia modernos es necesario analizar señales armónicas cuyo comportamiento es no lineal y no estacionario, por lo general estas señales son las que permiten el control de múltiples variables, como por ejemplo los indicadores de frecuencia media de interrupción por usuario en un periodo determinado y tiempo total promedio de interrupción por usuario en un periodo determinado (por sus siglas en ingles, SAIDI y SAIFI respectivamente), del cual hacen parte del marco legal colombiano regulado por la CREG (Comisión de regulación de energía y gas) y vigilado por la Superintendencia de servicios públicos domiciliarios en el marco de las leyes 142 y 143 de 1994 del congreso de la República. Estos indicadores se utilizan con el fin de detectar fallas en la red, indicar las contribuciones de los generadores distribuidos sobre el comportamiento dinámico del sistema de potencia, y de manera general en la optimización de la potencia distribuida (IEEE 2009; Kornatka 2017).

Una de las estrategias más comunes de uso en los sistemas de potencia para analizar señales ha sido la transformada Rápida de Fourier (FFT por sus siglas en inglés), pero una gran limitación es la resolución en el domino del tiempo de este método; por lo tanto, se necesitan nuevos métodos que permitan tener buena resolución tanto en el domino del tiempo como en el de la frecuencia, y además que puedan involucrar el concepto de frecuencia instantánea para una detección precisa y rápida de perturbaciones. Las transformadas de Hilbert-Huang, Wavelet y Wigner-Ville han surgido como una ayuda para resolver este problema (Gasca et al. 2018; Puliafito et al. 2017). La idea básica de un análisis tiempo-frecuencia es el diseño de una estrategia que pueda describir las características de una señal en ambos dominios, de 
manera que no solo sea posible detectar la presencia de cualquier componente frecuencial sino también el instante de tiempo en el que se presenta (Afroni et al. 2013).

La HHT es un método adaptativo para el análisis en ambos dominios el cual puede ser utilizado para trabajar sobre señales con el comportamiento previamente descrito, basado en el principio de frecuencia instantánea, buscando una detección precisa e inmediata de las perturbaciones. Al comparar la HHT con la transformada de Fourier, es posible afirmar que la primera tiene la habilidad de detectar patrones de comportamiento con mayor facilidad en señales con fuertes oscilaciones. El análisis mediante WT es bastante poderoso en señales no estacionarias (Bueno-Lopez et al. 2017), mientras la WVD ha demostrado mejor resolución que la FFT en diferentes aplicaciones. Usualmente cada una de estas estrategias ha sido utilizada de manera independiente y en algunas ocasiones se han visto aplicaciones donde se combinan buscando aprovechar los beneficios individuales de cada herramienta (Drummond and Sutanto 2010; Senroy et al. 2007).

Este documento se organiza de la siguiente manera: la primera sección presenta una revisión de la HHT, WT y la WVD con sus aplicaciones en sistema de potencia, realizando la síntesis de la información, en el cual se desarrolló un proceso de condensación de la base de datos, tomando el criterio de frecuencia instantánea como base fundamental. A partir del procedimiento descrito anteriormente, se procedió a realizar la depuración de la información, seleccionando la indicada para este tipo de análisis. En la segunda sección se desarrolló el análisis de cada uno de los tipos de métodos para detección de variaciones en sistemas de potencia con alta penetración de energía no convencional, y la comparación entre ellas, obteniendo las ventajas y desventajas de la aplicación de las técnicas según sea el caso específico.

\section{Análisis de señales no lineales y no estacionarias}

El análisis temporal de señales ha jugado un papel fundamental en la evolución de las diferentes estrategias que buscan extraer características de una serie de tiempo, sin embargo en sistemas no lineales y no estacionarios esto no es suficiente y se hace necesario recurrir también al análisis espectral. En muchas ocasiones se asume que los sistemas son estacionarios con el fin de simplificar el análisis pero esto restringe las posibilidades de conocer el comportamiento real de algunos sistemas dinámicos. Con base en lo anterior, es necesario hacer una revisión de algunos métodos que permiten tratar con este tipo de sistemas.

\subsection{Transformada de Hilbert-Huang (HHT)}

La HHT es un concepto presentado por Norden E. Huang en (Huang et al. 1998) y está compuesta de dos partes: Descomposición empírica de modos (EMD) y Espectro de Hilbert (HS). La EMD consiste en la descomposición de la señal original en varias señales, que permitan detectar diferentes componentes frecuenciales, llamadas IMFs (función de modo intrínseco), esta descomposición se debe desarrollar hasta encontrar una función monotónica que indica la incapacidad de extraer más IMFs. La IMF debe ser una función que cumpla dos condiciones: 1) el numero de extremos y el numero de cruces por cero debe ser diferente solo de uno, y 2) su media local es cero. Una vez terminada la descomposición se aplica la Transformada de Hilbert (HT) a cada una de las IMF que componen la señal original y con este proceso se obtiene el HS. El HS es una representación en 3D de la amplitud y frecuencia instantánea con respecto al tiempo. El principal problema del método es denominado la mezcla de modos (mode mixing en inglés) que consiste en la aparición de una misma componente frecuencial en diferentes IMF. Este problema se ha resuelto con la denominada señal máscara (masking signal en inglés). 


\subsubsection{EMD con máscara}

En 2005, en (Deering and Kaiser 2005) se define un método de mejora para la EMD, basado en el uso de señales máscara. Este algortimo busca eliminar o reducir la mezcla de modos o componentes frecuenciales que presenta el método convencional. El algoritmo se define de la siguiente manera:

- Se construye una señal máscara, $s(t)$, a partir de la información de la señal original, $x(t)$. Se consideran los valores de frecuencias que aparecen en varias de las IMFs para diseñar la máscara de manera que al ser añadida permita separar las componentes cercanas.

- Se realiza la $E M D$ a las dos siguientes señales, obtenidas a partir de la original y la mascara:

$$
\begin{aligned}
& x^{+}(t)=x(t)+s(t) \\
& x^{-}(t)=x(t)-s(t)
\end{aligned}
$$

- $\quad$ Posteriormente, se obtienen $I M F^{+}$para la señal $x^{+}(t)$ e $I M F^{-}$para la señal $x^{-}(t)$.

- Se obtiene una nueva IMF a partir de los resultados obtenidos y se define como:

$$
I M F(t)=\frac{I M F^{+}(t)+I M F^{-}(t)}{2}
$$

- Se aplica el proceso anteriormente descrito de manera iterativa aplicando cada iteracion al residuo resultante.

\subsection{Transformada Wavelet (WT)}

En 1980 fue desarrollada la transformada Wavelet (WT) por Morlet and Grossmam. La WT descompone una señal en bandas de frecuencia, que se generan por medio de una función llamada wavelets madre y operaciones de traslación de funciones en el dominio del tiempo, de modo que responde a las necesidades óptimas de resolución en una análisis de tiempo-frecuencia para todos los rangos de señales (O'Toole et al. 2008). En la actualidad la WT es un método popular dentro del análisis de señales de sonido y vibración en ingeniería. Además, posee una gran eficiencia y superioridad en la resolución como se muestra en (Mallat 1989).

\subsection{Distribución Wigner-Ville (WVD)}

La distribución de Wigner-Ville fue presentada por Wigner para investigar la mecánica cuántica en el año 1932 y aplicado en el procesamiento de señales por Ville 15 años después. Consiste en una representación en tiempo-frecuencia, evaluando una metodología de ventana deslizante de análisis de longitud-finita simétrica, que utiliza el conjugado de la ventana deslizante. La función de ventana deslizante debe cumplir las siguientes condiciones: 1) La condición inicial de la ventana deslizante es 0 , y 2) El modulo del tiempo-frecuencia a analizar debe ser mayor que la longitud en tiempo de la onda (Leonowicz 2000).

La Tabla 1 muestra las ventajas y desventajas de las metodologías del análisis en tiempo frecuencia. La FFT posee el problema de aliasing que consiste en la imposibilidad de descomponer una onda en una señal monotónica, siendo una estrategia de poca eficiencia para señales de onda no lineales y no estacionarias. La WT y WVD son similares a la FFT, al descomponer una señal en una serie de funciones básicas, pero con problemas distintos. Uno de los aspectos más críticos en la WT es la selección del tipo de wavelet (ventana) que más se coincida con la señal a analizar, con el fin de ajustarse a los componentes de bajas y altas frecuencias de la señal. 
Table 1. Comparación de las metodologías del análisis en tiempo-frecuencia

\begin{tabular}{c|cccc}
\hline & FFT & WVD & WT & HHT \\
\hline Base & Prioridad & Prioridad & Prioridad & Adaptativo \\
\hline No lineal & x & $\checkmark$ & x & $\checkmark$ \\
\hline No estacionaria & x & x & $\checkmark$ & $\checkmark$ \\
\hline Extracción & x & x & x & $\checkmark$ \\
de características & & & & \\
\hline
\end{tabular}

La WVD posee una mejor resolución en diferentes aplicaciones, pero al igual que la FFT no tiene la capacidad de descomponer una señal en señales monotónicas, por lo tanto, genera una distorsión del espectro de frecuencias.

Sin embargo, la HHT no está exenta de problemas, su debilidad está en la manera heurística de seleccionar el tipo de EMD, al igual que la WT depende de una función madre; aunque los resultados tiempo frecuencia poseen una mayor resolución y la capacidad de descomposición para cualquier tipo de señal, si no se selecciona la función base adecuada el resultado de las IMFs no será el indicado.

Tomando las características descritas, se requiere de una herramienta computacional que ensamble en la HHT las virtudes de las otras metodologías, con el fin de dejar a un lado la estrategia heurística, esta iniciativa se aborda parcialmente en (Sanabria-Villamizar et al. 2019).

\section{Análisis de señales en sistemas de potencia}

El comportamiento de las señales de un sistema de potencia eléctrico es no lineal y no estacionario, por eso es fundamental aplicar uno de los métodos descritos para evidenciar las perturbaciones del sistema. En (Puliafito et al. 2017) se muestra la actuación de consumo en potencia activa y reactiva de un restaurante, conjunto residencial, almacén de comida, comercializadora minorista y un local de servicios generales; donde se aplicó la HHT a la señal de los datos para identificar la variación instantánea en épocas del año, por tanto, se obtienen tendencias de consumo, para la planeación energética de la población.

La aplicación de la HHT puede detectar fallas en una red de distribución de energía eléctrica como se evidencia en (Bíscaro et al. 2015), (Sahani and Dash 2019) y (Alshahrani et al. 2016), al obtener la frecuencia y amplitud instantánea de una señal, teniendo la capacidad de percibir y clasificar cualquier variación (sag, swell, flicker, armónicos, notching, interrupciones, transitorios de frecuencia, etc.) en tiempo real con una eficiencia del 93.4\%. Con el propósito de realizar una corrección rápida en la red, garantizando la confiabilidad y la calidad de energía a los usuarios. En otros casos se puede emplear la HHT con técnicas de inteligencia artificial que permiten generar métodos de reconocimiento y clasificación en tiempo real, con la capacidad de actuación independiente en el sistema y una eficiencia del 95.3\%. Esta estrategia de análisis se expone en (Sahani and Dash 2018), donde muestra una poderosa herramienta computacional capaz de controlar las variaciones de la red. 


\section{Caso de Estudio}

\subsection{Señal sintética}

Con el objetivo de aclarar los conceptos presentados anteriormente, se presenta el análisis basado en la aplicación de la HHT con señal mascara y la Técnica Híbrida evidenciada en (Sanabria-Villamizar et al. 2019) sobre una señal sintética. La señal propuesta exhibe varios fenómenos: mezcla de modos y variación frecuencial.

La señal sintética se describe en la ecuación (1), (2) y (3), Además, se muestra en la Figura 1, con sus correspondientes mono-componentes frecuenciales.

$$
\begin{aligned}
& x(t)=5 \cdot \cos (2 \pi \cdot 200 t+20 \cdot \operatorname{sen}(2 \pi \cdot 60 t))+S_{1}+S_{2} \\
& S_{3}=\left\{\begin{array}{ccc}
10 \cdot \cos (2 \pi \cdot 50 t) & \text { si } & 0<t<0.05 \\
0 & \text { en otro caso }
\end{array}\right. \\
& S_{3}=\left\{\begin{array}{ccc}
10 \cdot \cos (2 \pi \cdot 100 t) & \text { si } & 0.05<t<0.01 \\
0 & \text { en otro caso }
\end{array}\right.
\end{aligned}
$$
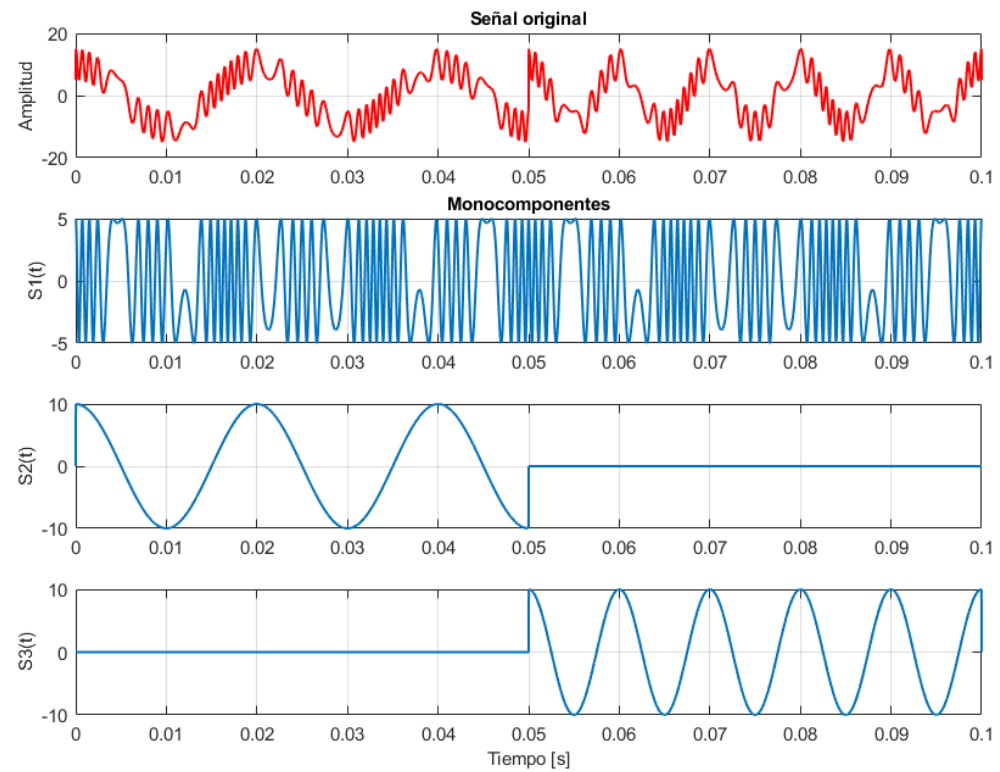

Figure 1. Señal original con sus respectivas frecuencias.

\section{Resultados de caso de estudio}

\subsection{Resultado de la HHT con señal mascara}

La descomposición empírica en modos se muestra en la Figura 2, donde en la primera IMF se observa una fuerte mezcla de modos entre las frecuencias de $200 \mathrm{~Hz}$ y $1000 \mathrm{~Hz}$, así mismo la siguiente IMF 
presentan mezcla de modos entre 5 y $100 \mathrm{~Hz}$. La tercera IMF presenta un comportamiento similar a la segunda IMF, finalmente en la cuarta queda la componente frecuencial de $50 \mathrm{~Hz}$. De igual forma en la Figura 3 se muestran las frecuencias instantáneas que corresponden a cada IMF.
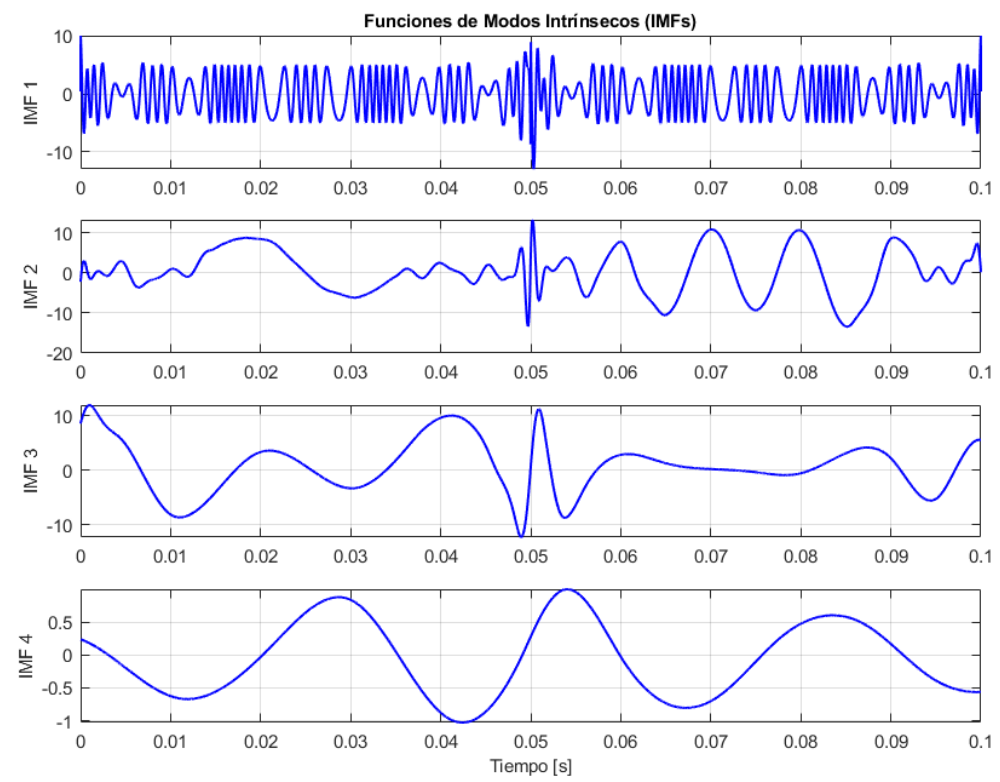

Figure 2. Descomposición usando EMD con señal mascara.
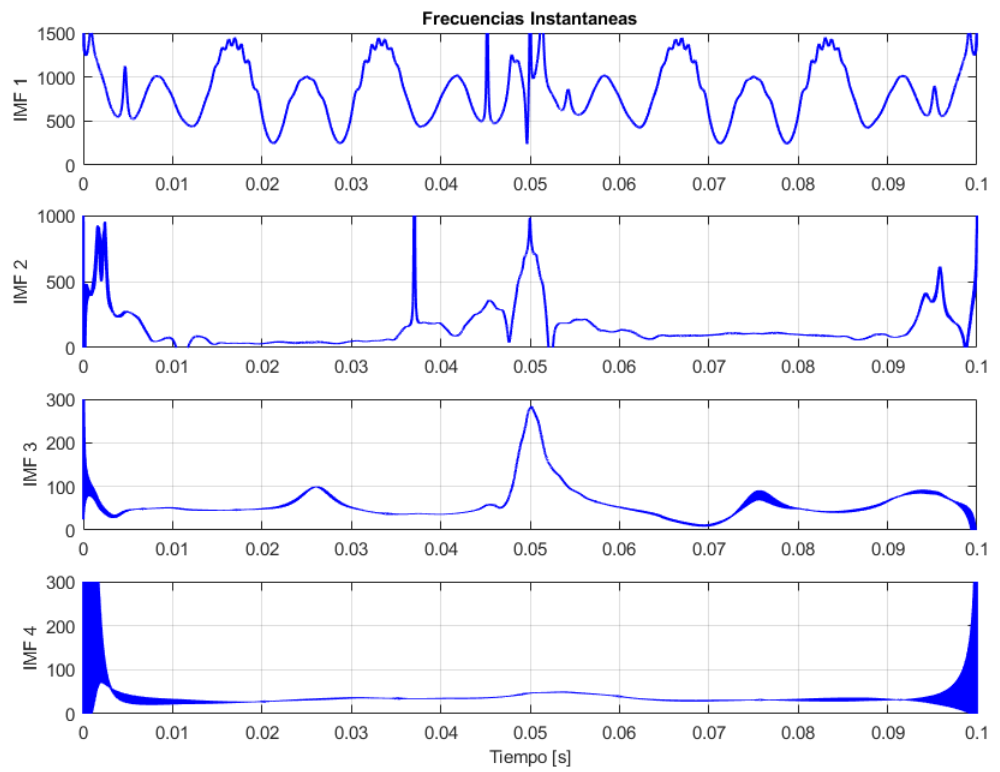

Figure 3. Frecuencia instantánea obtenida mediante EMD con señal mascara.

Cabe notar que al conocer la señal a analizar, se espera que en la descomposición de la señal sea visible la separación de los modos de frecuencia que componen la señal. Por esta razón se aplica la señal máscara con la forma $s(t)=a_{0} \operatorname{sen}(2 \pi f t)$, donde $a_{0}$ y $f$ se obtiene según Deering, como se muestra en (Deering and Kaiser 2005). En este caso la señal máscara que se utilizó fue $s(t)=35 \cdot \operatorname{sen}(2 \pi \cdot 410 t)$. 


\subsection{Resultado de la Técnica Híbrida}

Buscando una mejor descomposición de la señal de la Figura 1 se aplica la Técnica Híbrida presentada en (Sanabria-Villamizar et al. 2019). De forma concreta, la HT representa la combinación de la Transformada Rápida de Fourier, Transformada de Wavelet y Transformada de Hilbert-Huang. Los resultados de esta novedosa técnica se pueden observar en las Figuras 4 y 5, donde se muestran las IMFs obtenidas al realizar la Técnica Híbrida y las respectivas frecuencias instantáneas. De la Figura 4 se puede notar que se extraen de manera eficiente las componentes frecuenciales de la señal sintética. La primera y segunda IMFs representa el comportamiento del primer termino de la Ecuación 1. Por otra parte la tercera IMF muestra claramente la suma de las Ecuaciones 2 y 3 y para finalizar, la ultima IMF muestra parcialmente la frecuencia de $50 \%$. Las frecuencias instantáneas que representan el comportamiento de la cada una de las IMFs de la Figura 4, se muestran en la Figura 5.
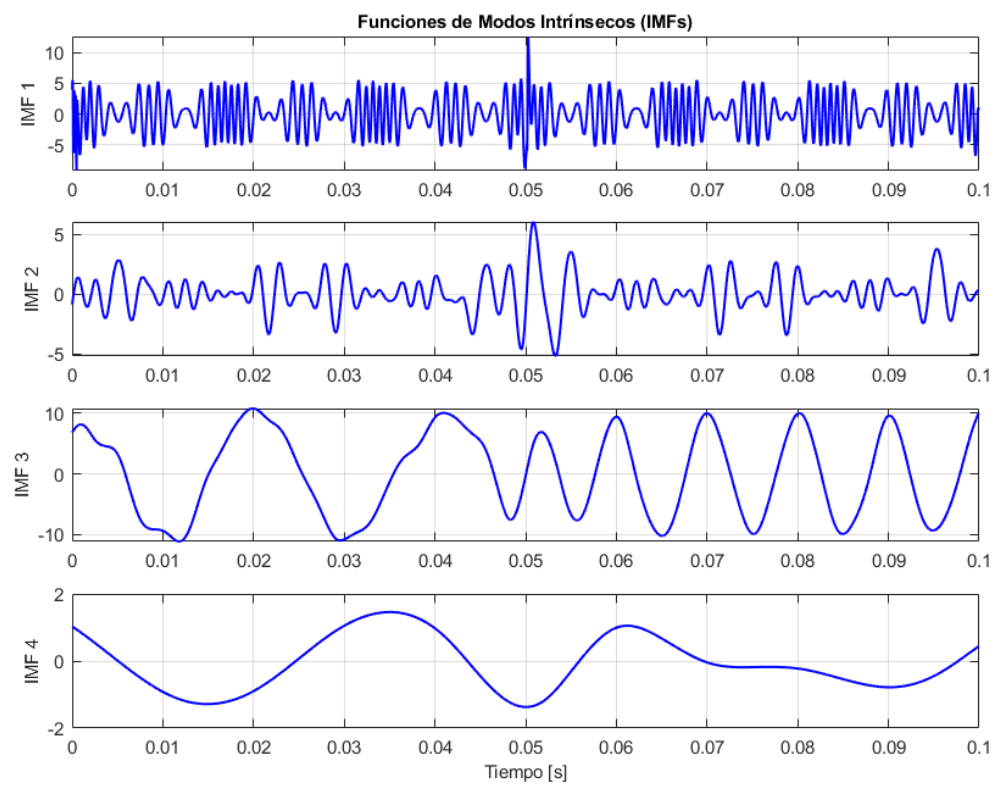

Figure 4. Descomposición usando la Técnica Híbrida.

Una de las formas de comprobar la efectividad, precisión y eficiencia en la descomposición de las señales es realizar la reconstrucción de las señales. Para constatar la validez de los resultados se realiza la reconstrucción del mono-componente 1 mostrado en la Figura 1. La comparación se realiza con respecto a la suma de la primera y segunda IMFs obtenidas en la Figura 4, dando como resultado una buena aproximación, con detalle lo exhibe la Figura 6.

De la misma manera se realiza la reconstrucción de los otros términos de la señal sintética, específicamente las Ecuaciones 2 y 3. Se muestra en la Figura 7 los componentes de $50 \mathrm{~Hz}$ y $100 \mathrm{~Hz}$.

\section{Conclusiones y Discusión}

En este documento hemos discutido las conceptos de importancia cuando se utilizan diferentes métodos de análisis de señales. En este caso, la Transformada de Hilber-Huang presenta dificultades como la mezcla de modos y el efecto de borde. El problema es detectado desde la implementación de la EMD con Señal Mascara. En la Figura 2 se observan las 4 IMFs obtenidas de la señal de estudio sintética. En la primera y segunda IMF se observa una mezcla de modos durante la toda la señal, proporcionada por el primer termino de la ecuación 1 que describe la señal sintética, donde se presenta una variación 

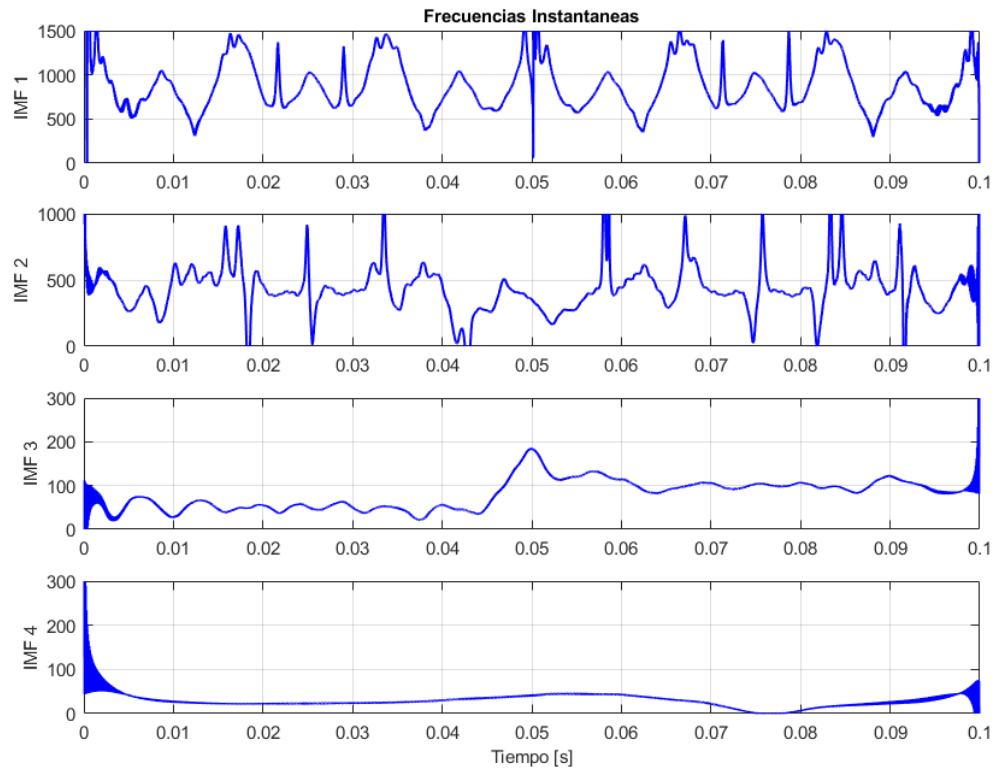

Figure 5. Frecuencia instantánea obtenida mediante la Técnica Híbrida.

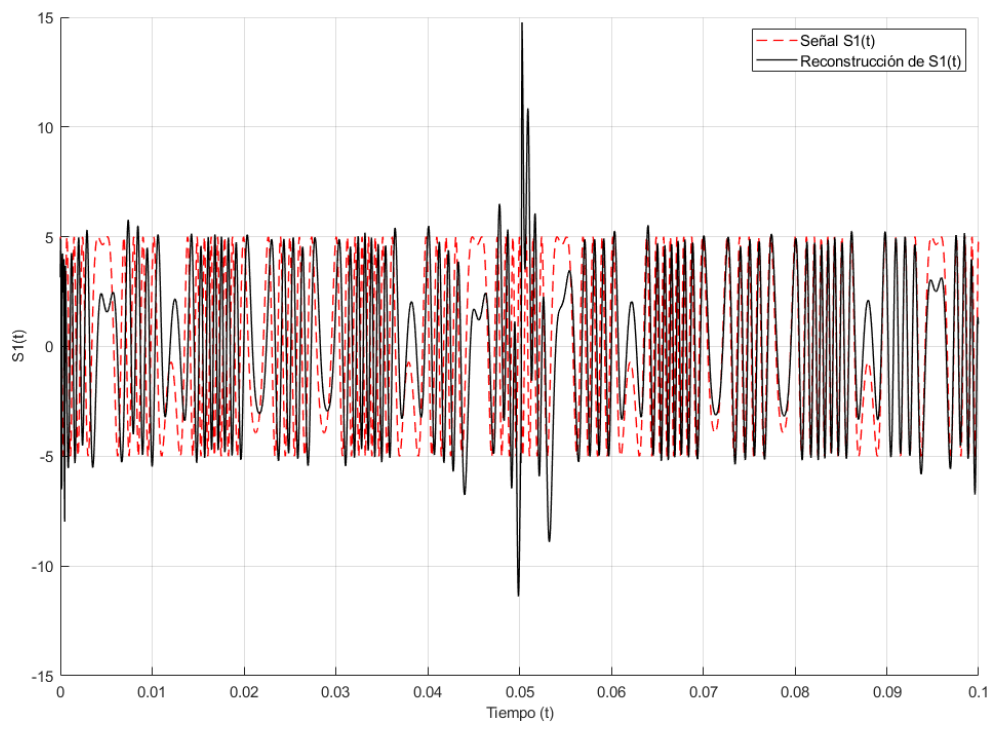

Figure 6. Reconstrucción de la Señal: $5 \cdot \cos (2 \pi \cdot 200 t+20 \cdot \operatorname{sen}(2 \pi \cdot 60 t))$.

de la fase. Este fenómeno es visible en la Figura 3 donde se observan las correspondientes frecuencias instantáneas. Con el objetivo de dar solución a este problema se realiza el análisis para la misma señal con una novedosa técnica. La Técnica Híbrida propone una estrategia más eficiente y rápida para el análisis de señales no lineales y no estacionarias, pero con una ventaja inigualable, tiene la capacidad de descomponer señales estocásticas y alto contenido de ruido, como la presentada en este documento. La Figura 4 permite evidenciar sin dificultad como en cada una de las IMF obtenidas se observan e identifican cada mono-componente de la señal a analizar.

El procedimiento de la HHT es efectivo para señales con sin ruido, con frecuencias lejanas entre si y con una fase constante. Por otra parte, la Técnica Híbrida emerge como una alternativa para aquellas señales con todas los antónimos de las características mencionadas anteriormente. Para complementar, 


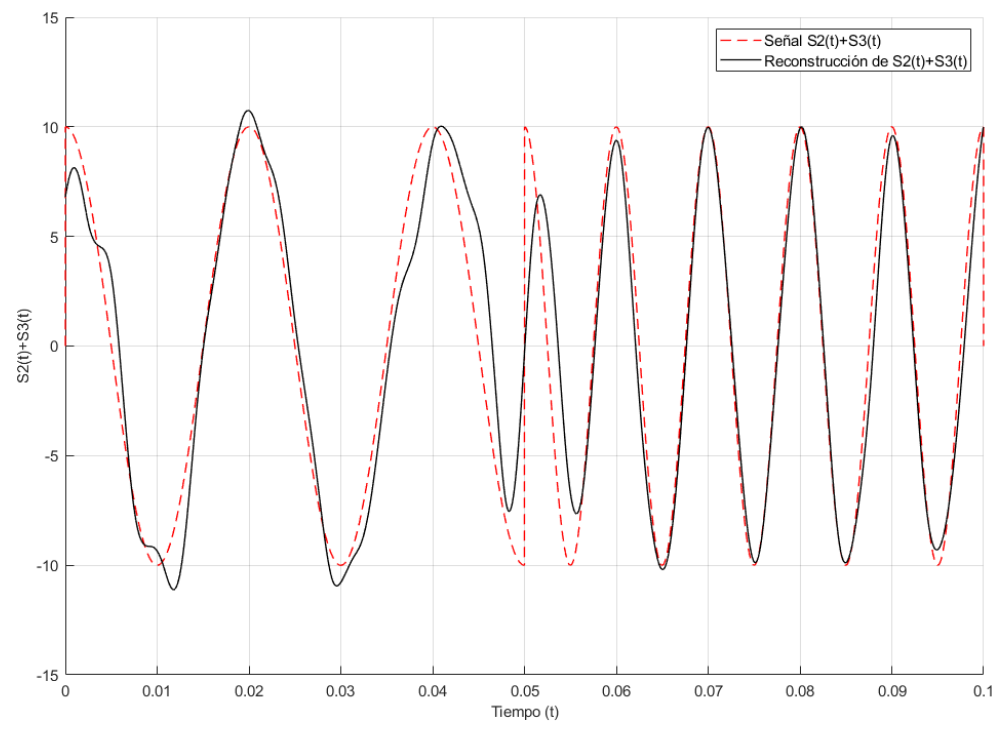

Figure 7. Reconstrucción de la Señal $S_{1}+S_{2}$.

ambas metodologías son eficaces para analizar señales que se conoce a priori su valor de frecuencia pero no su amplitud, los resultados obtenidos, los cuales se presentan en este articulo, dan inicio a una nueva estrategia para el análisis de señales.

Author Contributions: Conceptualización, J.B.-L. and J.M.S.-V; Metodología, J.B.-L. and J.M.S.-V.; Investigación, J.B.-L. and J.M.S.-V.; Escritura, revisión y Edición, J.B.-L. and J.M.S.-V.

\section{References}

Afroni, M. J., Sutanto, D., and Stirling, D. (2013). Analysis of nonstationary power-quality waveforms using iterative hilbert huang transform and sax algorithm. IEEE Transactions on Power Delivery, 28(4):2134-2144.

Alshahrani, S., Abbod, M., and Taylor, G. (2016). Detection and classification of power quality disturbances based on hilbert-huang transform and feed forward neural networks. In 2016 51st International Universities Power Engineering Conference (UPEC), pages 1-6. IEEE.

Anzalchi, A., Sundararajan, A., Moghadasi, A., and Sarwat, A. (2019). High-penetration grid-tied photovoltaics: Analysis of power quality and feeder voltage profile. IEEE Industry Applications Magazine, 25(5):83-94. doi:10.1109/MIAS.2019.2923104.

Bíscaro, A., Pereira, R., Kezunovic, M., and Mantovani, J. (2015). Integrated fault location and power-quality analysis in electric power distribution systems. IEEE Transactions on power delivery, 31(2):428-436.

Bueno-Lopez, M., Molinas, M., and Kulia, G. (2017). Understanding instantaneous frequency detection: A discussion of Hilbert-Huang Transform versus Wavelet Transform. In International Work-Conference on Time Series Analysis-ITISE, volume 1, pages 474-486, Granada, Spain. University of Granada.

Deering, R. and Kaiser, J. F. (2005). The use of a masking signal to improve empirical mode decomposition. In Proceedings.(ICASSP'05). IEEE International Conference on Acoustics, Speech, and Signal Processing, 2005., volume 4, pages iv-485. IEEE.

Drummond, C. F. and Sutanto, D. (2010). Classification of power quality disturbances using the iterative hilbert huang transform. In Proceedings of 14th International Conference on Harmonics and Quality of Power - ICHQP 2010, pages 1-7. doi:10.1109/ICHQP.2010.5625326.

Fan, Z. and Liu, X. (2012). A novel universal grid voltage sag detection algorithm. In 2012 Power Engineering and Automation Conference, pages 1-4. IEEE. 
Gasca, M. V., Bueno-Lopez, M., Molinas, M., and Fosso, O. B. (2018). Time-frequency analysis for nonlinear and non-stationary signals using hht: A mode mixing separation technique. IEEE Latin America Transactions, 16(4):1091-1098. doi:10.1109/TLA.2018.8362142.

Golpîra, H. and Messina, A. R. (2018). A center-of-gravity-based approach to estimate slow power and frequency variations. IEEE Transactions on Power Systems, 33(1):1026-1035. doi:10.1109/TPWRS.2017.2710187.

Huang, N. E., Shen, Z., Long, S. R., Wu, M. C., Shih, H. H., Zheng, Q., Yen, N.-C., Tung, C. C., and Liu, H. H. (1998). The empirical mode decomposition and the hilbert spectrum for nonlinear and non-stationary time series analysis. Proceedings of the Royal Society of London. Series A: Mathematical, Physical and Engineering Sciences, 454(1971):903-995. IEEE (2009). IEEE recommended practice for monitoring electric power quality. IEEE Std 1159-2009 (Revision of IEEE Std 1159-1995), pages c1-81. doi:10.1109/IEEESTD.2009.5154067.

Kavitha, V. and Subramanian, K. (2017). Investigation of power quality issues and its solution for distributed power system. In 2017 International Conference on Circuit, Power and Computing Technologies (ICCPCT), pages 1-6. doi:10.1109/ICCPCT.2017.8074372.

Kornatka, M. (2017). Distribution of saidi and saifi indices and the saturation of the mv network with remotely controlled switches. In 2017 18th International Scientific Conference on Electric Power Engineering (EPE), pages 1-4. doi:10.1109/EPE.2017.7967243.

Kumar, D. and Zare, F. (2016). Harmonic analysis of grid connected power electronic systems in low voltage distribution networks. IEEE Journal of Emerging and Selected Topics in Power Electronics, 4(1):70-79. doi:10.1109/JESTPE.2015.2454537.

Leonowicz, Z. (2000). Analysis of non-stationary signals in power systems using wigner transform and min-norm method. In 7th EEEIC Conference on Environment and Electrical Engineering, pages 43-46.

Liu, Z., Cui, Y., and Li, W. (2015). A classification method for complex power quality disturbances using eemd and rank wavelet svm. IEEE Transactions on Smart Grid, 6(4):1678-1685. doi:10.1109/TSG.2015.2397431.

Mallat, S. G. (1989). A theory for multiresolution signal decomposition: the wavelet representation. IEEE Transactions on Pattern Analysis E Machine Intelligence, (7):674-693.

Naderi, Y., Hosseini, S. H., Ghassemzadeh, S., Mohammadi-Ivatloo, B., Savaghebi, M., Vasquez, J. C., and Guerrero, J. M. (2020). Chapter 4 - Power quality issues of smart microgrids: applied techniques and decision making analysis. In Aleem, S. H. A., Abdelaziz, A. Y., Zobaa, A. F., and Bansal, R., editors, Decision Making Applications in Modern Power Systems, pages 89 - 119. Academic Press. doi:https:/ / doi.org/10.1016/B978-0-12-816445-7.00004-9.

O'Toole, J. M., Mesbah, M., and Boashash, B. (2008). A new discrete analytic signal for reducing aliasing in the discrete wigner-ville distribution. IEEE Transactions on Signal Processing, 56(11):5427-5434.

Puliafito, V., Vergura, S., and Carpentieri, M. (2017). Fourier, wavelet, and hilbert-huang transforms for studying electrical users in the time and frequency domain. Energies, 10(2). doi:10.3390/en10020188.

Sahani, M. and Dash, P. (2019). Fpga-based online power quality disturbances monitoring using reduced-sample hht and class-specific weighted rvfln. IEEE Transactions on Industrial Informatics.

Sahani, M. and Dash, P. K. (2018). Automatic power quality events recognition based on hilbert huang transform and weighted bidirectional extreme learning machine. IEEE Transactions on Industrial Informatics, 14(9):3849-3858.

Sanabria-Villamizar, M., Bueno-López, M., Molinas, M., and Bernal, E. (2019). Hybrid technique for the analysis of non-linear and non-stationary signals focused on power quality. In 2019 FISE-IEEE/CIGRE Conference-Living the energy Transition (FISE/CIGRE), pages 1-6. IEEE.

Senroy, N., Suryanarayanan, S., and Ribeiro, P. F. (2007). An improved hilbert-huang method for analysis of time-varying waveforms in power quality. IEEE Transactions on Power Systems, 22(4):1843-1850. doi:10.1109/TPWRS.2007.907542.

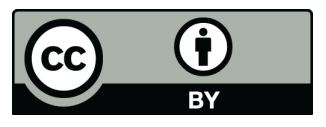

(C) 2020 by the authors. Licensee TESEA, Cartagena, Colombia. This article is an open access article distributed under the terms and conditions of the Creative Commons Attribution (CC BY) license (http:/ / creativecommons.org/licenses/by/4.0/). 